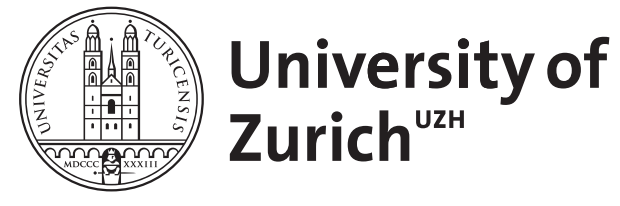

\title{
Sieben Thesen zum Begriff der âventiure
}

Schnyder, Mireille

Posted at the Zurich Open Repository and Archive, University of Zurich

ZORA URL: https://doi.org/10.5167/uzh-93726

Book Section

Published Version

Originally published at:

Schnyder, Mireille (2006). Sieben Thesen zum Begriff der âventiure. In: Dicke, Gert; Eikelmann, Manfred; Hasebrink, Burkhard. Im Wortfeld des Textes : worthistorische Beiträge zu den Bezeichnungen von Rede und Schrift im Mittelalter. Berlin: Walter de Gruyter GmbH Co. KG, 369-375. 


\section{MiREILLE SCHNYDER}

\section{Sieben Thesen zum Begriff der âventiure}

The author outlines the close relationship of the concept of âventiure to the act of telling a story. Initially, a coherent order of the apparent contingency of events could retrospectively be established at the end of the story by the âventiure, then the gradual separation of the aventiure from the body resulted in a confusing variety of possibilities for fate, which can be regarded as the beginning of modern era. Adventurers increasingly became artists of the word speculating with possibilities. Therefore, the term could also be applied to the early speculators and shareholders. Consequently, the âventiure which retrospectively establishes a coherent order comes to an end with the rise of the calculus of probabilities which made certainty possible from the beginning.

\section{These I:}

Aventiure gehört in den Bereich der Imagination und meint das in der Artifizialität der (Nach)Erzählung konstituierte Geschick.

Die französische avanture, als Ereignis, als Begebenheit, wird durch die bele conjointure bei Chrétien' zum Schicksalsfaden gereiht, der in der deutschen Artusliteratur dann, als der aventiure meine ${ }^{2}$, das erzählte Leben des Helden bestimmt. Aventiure ist somit in erster Linie und von Anfang an in der deutschen Literatur des Mittelalters die sinnstiftende Geschichte, die das Geschick des Einzelnen durch die Artifizialität der Nacherzählung konstituiert. ${ }^{3}$

Am Anfang des Iwein von Hartmann von Aue wird dies in der Erzählung Kalogrenants exemplarisch vorgefuihrt ${ }^{4}$ : Erst durch das retrospektive Erzählen

1 Les romans de Chrétien des Troyes, Bd. 1: Erec et Enide. Hrsg. von Mario RoQues, Paris 1978 (Les Classiques Français du Moyen Âge 80), hier V. 14.

2 Gottfried von Straßburg: Tristan und Isold. Hrsg. von FrJEDRICH RANKE, Berlin 1959, hier V. 4627.

3 Anders als eine immer wieder geäußerte Meinung, dass mit dem Sieg die âventiure nicht mehr existiert, meine ich, dass erst durch den Sieg und das dadurch ermöglichte Erzählen auf diesen Sieg hin, die âventiure sich konstituiert. Vgl. zur sich auflösenden âventiure $u$. a. mit entsprechenden Verweisen auf weitere Forschungsliteratur: KLAUSPETER WEGERA: „mich enhabe diu âventiure betrogen“. Ein Beitrag zur Wort- und Begriffsgeschichte von âventiure im Mittelhochdeutschen. In: Das Wort. Seine strukturelle und kulturelle Dimension. Festschrift für Oskar Reichmann zum 65. Geburtstag. Hrsg. von VILMOS ÁGEL u. a., Tübingen 2002, S. 229-244, hier S. 238.

4 Hartmann von Aue: Iwein. Text der siebenten Ausgabe von GEORG FRIEDRICH BENECKE/KARL LACHMANN/LUDWIG WOLFF. Übersetzung und Nachwort von THOMAS 
am Artushof, im Kreis der Ritter, wird die Suche nach etwas Unbekanntem, Unerhörtem in Raum und Zeit gegliedert, benannt und hörbar gemacht. Damit wird das Unerhörte und als wunderbar Begegnende des Erlebnisses in der retrospektiven Erzählung zum Ende einer Geschichte, von dem her sich - im Blick zurück - das Vorher ordnet. ${ }^{5}$ Aventiure meint also das in der Sprache geformte Erleben, das dadurch in eine Gedankenstruktur und eine Sinnstruktur eingebaut wird und entsprechend in den Vorstellungsraum und die imaginären Welten eintritt. Deshalb kann es auch die âventiure, die richtig gefügte Geschichte eines Helden sein, die dem Erzähler als Vorlage für sein neues Erzählen dient. Es gibt keine âventiure, die nicht erzählt ist. ${ }^{6}$

\section{These II:}

Aventiure ist Kontingenzbewältigung, ist Sinngebung (für das Mittelalter besser: Sinn-Offenlegung) für die Welt.

Die Setzung und Ordnung der Welt durch die Sprache ist Realisierung eines Geschicks, das, als göttlicher Plan erkennbar, sich in der Erzählung abbildet. Als ein in eine Sinnstruktur hineinerzähltes Erlebnis ist âventiure immer Instandstellung eines aus der Ordnung geratenen Geschehens und die Wiederherstellung der Welt. Die âventiure-Erzählung am Hof von Artus rückt die aus den Fugen geratene Welt wieder zurecht. Es muss erzählt werden, was geschehen ist, um das Geschehene in die Macht- und Kulturordnung des Hofes einzugliedern, aber auch, um die von Gott gegebene âventiure, als Geschick und Geschichte, offenbar zu machen.

Im Erec Hartmanns von Aue ist deutlich, dass die âventiure von Gott kommen muss': Erec reitet am Anfang des Romans Iders $\hat{u} f$ âventiure nâch (V. 221), um sich - sofern Gott ihm die Möglichkeit gibt (V. 490f.) - für die von Iders erlittene Schmach zu rächen. In dieser Hoffnung, $\hat{u} f$ selcher âventiure wân (V. 492), bricht er auf. Aventiure wird hier zur Erfüllung und Einlösung

CRAMER. 4., überarb. Aufl., Berlin, New York 2001.

5 Wenn VOLKER MERTENS (in seinem Beitrag zum vorliegenden Band, S. 339-346, hier S. 342) meint, dass âventiure, als Aufgabe des Ritters zum Kampf, durch den Sieg wieder Ordnung herstellt, fallt das letztlich genau mit dieser Zuspitzung des âventiureBegriffs auf das Ende einer Geschichte zusammen. Denn von da her, von der letzten großen Bewährung und dem letzten großen Sieg des Ritters, wird die âventiure in ihrer Sinnhaftigkeit, das heißt auch Geordnetheit, erschlossen.

6 Damit wird der semantische Kern des französischen Begriffs (eine , e r zählens w e r t e Begebenheit') in der deutschen Rezeption des Begriffs verstärkt; vgl. dazu den Beitrag von FRANZ LEBSANFT in diesem Band (S. 311-337, hier S. 330, Sperrung von mir, M. S.).

7 Erec von Hartmann von Aue. Hrsg. von ALBERT LEITZMANN, fortgef. von LUDWIG WOLFF, 6. Aufl. besorgt von CHRISTOPH CORMEAU/KURT GäRTNER, Tübingen 1985 (ATB 39). 
eines göttlichen Plans. Der göttliche Ursprung von âventiure wird im Wilhelm von Österreich von Johann von Würzburg dann explizit gemacht, wenn Gott als schepfer aller aventiur (V. 2435) angesprochen wird. ${ }^{8}$

Deshalb verbindet sich der Begriff der âventiure nicht nur mit dem Zufallsgeschehen der Fortuna, sondern eben auch mit vrou Sielde, als dem göttlichen und providentiellen Heil.

Entsprechend werden die Schluss-âventiure der Artusromane als das dem Helden zugedachte Geschick erkannt. Das Schlussabenteuer von Erec ist s e in e âventiure, wozu vrou Scelde ihm die notwendige Aussteuer mitgegeben hatte (V. 9899-902).

Die Setzung und Ordnung der Welt durch die Sprache ist Realisierung eines Geschicks, das - im Verstehensraum des Mittelalters - ein göttlicher Plan ist, der sich in der Erzählung abbildet. Es ist die salde, aus der heraus sich zum Schluss eine Geschichte als sinnvolles Gefüge, als âventiure, erzählen lässt. Aventiure ist also das Ereignis, die Begebenheit, von der aus sich im Rückblick alle Zufälligkeiten und Ereignisse in ein sinnvolles Gefüge lesen lassen. Die Suche nach diesem Ordnungspunkt in der verwirrenden Ereignisfülle des Geschehens findet ein schlagendes Bild in der Metaphorik des Glücksspiels (Würfelspiels).

These III:

Aventiure ist das Glück des Spiels.

Findet sich in der gliedernden und ordnenden âventiure-Erzählung die Sinngebung für das einzelne, sinnopake Ereignis, ist die âventiure das Glück, das in den einzelnen Würfen des Zufallsspiels verborgen liegt. Für den in der Welt verstrickten Helden ist es das Fortunaglück der vergänglichen Zufälligkeit; aus der Perspektive des zurückblickenden und die Einzelereignisse ordnenden Erzählers aber ist es das als Geschick im Geschehen wirkende salde-Glück göttlicher Vorsehung.

Das wird schon deutlich im Erec, wenn dieser die große Schluss-âventiure als seine âventiure erkennt, als das ihm bestimmte salic spil (V. 8538), als ein wunschspil (V. 8530). Großartig ausgeführt ist es aber bei Wolfram von Eschenbach im Parzival. ${ }^{9}$ Hier ist es aventiure, die den kleinen Helden in die Erzählung wirft - wie einen Würfel ins Spiel (V.112,9f.). Erkennt Erec am

8 Johanns von Würzburg Wilhelm von Österreich. Aus der Gothaer Handschrift hrsg. von ERNST REGEL, Berlin 1906, Nachdruck Dublin, Zürich 1970 (DTM 3).

9 Wolfram von Eschenbach: Parzival. Nach der Ausg. KARL LACHMANNs revidiert und kommentiert von Eberhard NellmanN. Übertragen von DiETER KüHN, 2 Bde., Frankfurt a. M. 1994 (Bibliothek des Mittelalters 8/1-2). Dazu: MireiLle SCHNYDER: Glücksspiel und Vorsehung. Die Würfelspielmetaphorik im Parzival Wolframs von Eschenbach. In: ZfdA 131 (2002), S. 308-325. 
Schluss seiner Weltreise im Kampf gegen Mabonagrin das ihm zugedachte Spiel, von dem aus sein ganzes Irren Sinn erhält und erzählbar wird, ist im Parzival mit dem ersten Würfelwurf der âventiure, der Geburt Parzivals, auch seine Erzählung bestimmt. Die âventiure ist es, die Parzival in undurchschaubarer Konsequenz durch die Wirnisse der Welt dahin bringt, wo ihn die scelde hingedacht hatte, wie es zum Schluss heißt: dar sîn doch saelde het erdâht (V. 827, 18).

These IV:

Aventiure ist das Produkt eines Erkenntnisprozesses

und somit eine Gedankenfigur.

Aventiure erschließt sich als saelic spil nur demjenigen, der richtig fragt, und ist somit Ende eines Erkenntnisprozesses. Aventiure suchen können viele, âventiure finden nur diejenigen, die richtig fragen und mit dem Herzen wahrnehmen. Es ist die Fähigkeit, die Ereignisse der Welt auf sein eigenes Geschick hinzuordnen und die eigene Existenz in ein Sinngefüge hineinzudenken.

Deutlich wird dies am Schluss des Erec, wenn dieser nach langem Fragen die ihm zugedachte âventiure erkennt und besteht, während die auf dem Zaun aufgespießten Köpfe all seiner Vorgänger Zeugnis ablegen davon, dass diese nur mit tumbe $[m]$ herze die âventiure suchten (V. 8480f.). Es ist das richtige Verstehen (d. h. ein Hören im Herzen), das zur âventiure befähigt. Deshalb klopft die (personifizierte) Aventiure im Parzival auch an die Herztür des Erzählers: ,Tuot ûf.' (V. 433,1). Und auch Gawein findet den Weg zu Clinschors Burg und damit zu seiner âventiure nur durch sein eindringliches Fragen im Haus des Fährmanns (V. 554,23-555,13).

\section{These V:}

Die Vervielfältigung der âventiure-Erzählungen wird zum Problem für die Erkenntnis des Einzelgeschicks.

Und das ist als Anfang der Moderne zu sehen.

Aventiure, als sich in der Imagination konstituierendes Geschick, Ergebnis eines Erkenntnisprozesses, der hinter dem Zufall die göttliche Fügung sehen lehrt, löst sich durch die Vervielfältigung der in den Erzählungen gebotenen Geschick-Möglichkeiten immer mehr vom Körper des Einzelnen und entsprechend von dem nur ihm bestimmten Geschick.

Begegnet der Held anfangs unerhörten Begebenheiten, die in der retrospektiven Erzählung dann mit Sinn versehen werden müssen, ist es im Verlauf der Vervielfältigung der erzählten Geschichten immer mehr die schon erzählte aventiure, die begegnet. Und es geht darum, dass der Held in einer Welt der 
Geschichtenwirrnis seine eigene Geschichte lesend konstruiert. Die reitende Suche nach aventiure im Wald von Breziljan, als dem letztlich von Gott vorbestimmten eigenen Geschick, wird zur erlesenen Suche nach aventiure im Textwald schon erzählter Geschichten. Und es ist nicht mehr Gott, der hier Glück und Unglück gibt, sondern die in den Geschichten angebotenen Lebensmuster. $^{10}$

Im Wilhelm von Österreich meint der Held in dem Hauptmann Aventiur denjenigen erkennen zu können, der Glück und Unglück gibt, Tod und Ruhm (V. 3332-43) und nennt ihn zarter Got (V. 3373). Ein Missverständnis, das da deutlich wird, wo der Aventiur den Helden zurechtweist, als der ihm gesteht, dass seine âventiure-suoche nichts anderes als Todessucht ist, da er vom Glück verlassen sei (V. 3380-83). Hier verweist der Aventiur auf Gott, der den Helden bis hierher getragen hätte und also auch nicht im Stich lassen werde (V. 3390f.).

Die Schwierigkeit der âventiure-Suche des Helden aber ist, dass die Welt, in die er hineinreitet, eine Welt der Texte ist, die der Erzähler als, Ährenleser aus seinen Vorgängern zusammenstellt.

Es ist nicht von ungefähr, dass Grimmelshausen an den Anfang seines Simplicissimus ein Titelkupfer stellt, das das Konterfei dieses Hauptmanns Aventiur ist: das Buch wird zum Ort des Abenteuers, die Lektüre wird zum Abenteuer."

Diese Welt der Geschichtenwirmis, hinter der nur noch mit Zwang und Mühe eine leitende göttliche Instanz gesehen werden kann, ist als Anfang der Moderne $\mathrm{zu}$ sehen. ${ }^{12}$

10 Zur Vermittlung vorrangig literarischer Erfahrung über das Abenteuer in den spätmittelalterlichen Romanen vgl. KLAUS RIDDER: Mittelhochdeutsche Minne- und Aventiureromane. Fiktion, Geschichte und literarische Tradition im späthöfischen Roman: Reinfried von Braunschweig, Wilhelm von Österreich, Friedrich von Schwaben, Berlin, New York 1998 (Quellen und Forschungen zur Literatur- und Kulturgeschichte 12), S. 30 .

11 Vgl. dazu Paul MiCHEL: Eine bisher unbeachtete Vorlage für das Titelkupfer des Simplicissimus: der abenteür hauptman. In: Simpliciana 8 (1986), S. 97-109; MIREILLE SCHNYDER: âventiure? waz ist daz? Zum Begriff des Abenteuers in der deutschen Literatur des Mittelalters. In: Euphorion 96 (2002), S. 257-272, hier S. 271.

12 Als Beispiel für diesen Prozess führt MiCHEl FouCAult (Die Ordnung der Dinge. Eine Archäologie der Humanwissenschaften, Frankfurt a. M. 1974, hier S. 81) Don Quijote an: „Don Quichotte ist das erste der modernen Werke, da man darin die grausame Vernunft der Identitäten und Differenzen bis ins Unendliche mit den Zeichen und den Ähnlichkeiten spielen sieht. Die Sprache zerbricht darin ihre alte Verwandtschaft mit den Dingen, um in jene einsame Souveränität einzutreten, aus der sie in ihrem abrupten Sein erst als zur Literatur gewordene wieder erscheinen wird. Die Ähnlichkeit tritt dort in ein Zeitalter ein, das für sie dasjenige der Unvernunft und der Imagination ist. Wenn die Ähnlichkeit und die Zeichen einmal losgeknüpft sind, können zwei Erfahrungen sich konstituieren und zwei Personen in ihrer Gegenüberstellung erscheinen." 


\section{These VI:}

Das Abenteuer löst sich immer mehr vom Körper des Helden.

Die vom Körper losgelöste Potenz des Wortes wird zum Träger des Abenteuers, während der Abenteurer hinter dem Ofen sitzen bleibt (wie Schelmuffsky bei Christian Reuter ${ }^{13}$ ), oder sich als Gaukler und Vagabund durch erfundene Geschichten eine abenteuerliche Existenz zulegt. So ist der Begriff des Abenteurers im 15. und 16. Jahrhundert gern für das mittelhochdeutsche spilman gebraucht und ist immer mit Betrug und Täuschung in Verbindung gebracht: Abenteurer machen den Leuten etwas vor durch Erzählungen oder Possen. ${ }^{14}$ Wird hier einerseits der hinter dem Ofen sitzende Leser zum âventiuraere, in dessen imaginären Erlebnissen sich das Abenteuer ganz vom Körper löst, $\mathrm{zu}$ einem Hirngespinst wird und da eine neue Welt schafft, ist es nicht verwunderlich, dass mit dem Aufkommen der Geldgeschäfte, der Spekulationen und auf imaginäre Werte setzenden Investitionen, für deren Einsatz der Körper keine Rolle mehr spielt, der Begriff sich von der sprachlichen Imagination, der fiktionalen Erlebniswelt in die Welt der Geldgeschäfte überträgt. Seit dem 15. Jahrhundert ist es der Glücksspieler, der als adventurer bezeichnet wird, und in der frühneuzeitlichen Geldwirtschaft wird der Begriff auf den Spekulanten und den Aktionär übertragen. ${ }^{15}$

Das Spiel mit den Worten in der Erzählung als Konstituierung einer Wirklichkeit wird gleichgesetzt mit dem riskanten Einsatz von Geld. Wortgeschäfte und Geldgeschäfte, als vom Körper losgelöste Einsatz- und Erlebnisfelder, ganz an den Vorstellungsraum gebunden, begegnen sich im Begriff des Abenteuers. $^{16}$

13 Christian Reuter: Schelmuffskys warhafftige curiöse und sehr gefährliche Reisebeschreibung zu Wasser und Lande. Hrsg. von ILSE-MARIE BARTH, Stuttgart 1992 (RUB 4343).

14 Vgl. dazu WeRner WelzIG: Der Wandel des Abenteurertums. In: Pikarische Welt. Schriften zum europäischen Schelmenroman. Hrsg. von HELMUT HEIDENREICH, Darmstadt 1969 (WdF 163), S. 438-454 (zuerst in: Ders.: Beispielhafte Figuren. Tor, Abenteurer und Einsiedler bei Grimmelshausen, Graz, Köln 1963, S. 100-114).

15 Vgl. dazu: The Oxford English Dictionary. Second Edition. Hrsg. von JoHN A. SIMPSON/EDMUND S. C. WEINER, Oxford 1989, Bd. 1, S. 187a (,adventure' Pkt. 7) u. S. 187b-c (,adventurer' Pkt. 1-4).

16 Wenn Daniel Defoe zur gleichen Zeit wie Robinson Crusoe (1719), (,the life and adventures", wie es im Untertitel heißt), anonym einen Traktat The Gamster (Der Spieler) publiziert, in dem er dem Zielpublikum der ,adventurers“ an Börse und Lotterie Grundsätze der Wahrscheinlichkeit zu vermitteln sucht, lässt das aufhorchen. Im Englischen wird seit 1625 ein Geldrisiko, eine Investition, eine Spekulation, eine kommerzielle Unternehmung mit adventure bezeichnet. Adventurers werden denn auch diejenigen genannt, die - im Gegensatz zu den auswandernden Pflanzern - in Übersee-Companien (Gesellschaften) investieren. Vgl. dazu wie Anm. 15 sowie: Encyclopédie ou Dictionnaire raisonné des sciences des arts et des métiers. Hrsg. von Denis Diderot, Paris 175180, Nachdruck Stuttgart-Bad Cannstatt 1966, Bd. 1, S. 868b-869a. 


\section{These VII:}

Die Sinngebung vom Ende her wird durch eine Versicherung vom Anfang her abgelöst. Die âventiure wird so zum kalkulierbaren Risiko.

Während das als Glücksspiel wahrgenommene Stolpern des aventiuraere durch göttliche Fügung vom Ende her als saeldenspil wahrgenommen werden kann, wird das vom Körper losgelöste Abenteuer durch die Wahrscheinlichkeitsrechnung dann seit dem 18. Jahrhundert vom Anfang her zu sichern gesucht. Hatte vorher der Erzähler die Wahrscheinlichkeit des Geschehens zu bündeln, die Zufälligkeiten des Erlebens durch die Erzählung in die Wahrscheinlichkeit, als eine auf Wahrheit hin durchsichtige Ordnung zu bringen, ist es nun die neu entdeckte Wahrscheinlichkeitsrechnung, die den Zufall in die Berechenbarkeit bringt.

Damit löst sich aber auch der Begriff der âventiure auf. Mit dem Versicherungswesen, das sich bezeichnenderweise im Umfeld der adventurer, der Seekaufleute, herausgebildet hat, endet das Abenteuer. So findet sich in der Encyclopédie von Diderot (1751-80) unter dem Stichwort ,aventure' nicht nur der Eintrag: „évenement extraordinaire ou surprenant, soit réel soit imaginaire" mit dem Verweis auf "fable“, sondern auch der Eintrag aus dem Bereich des Handels (commerce): ,mettre de l'argent à la grosse aventure, c'est le placer sur un vaisseau, où l'on court risque de le perdre par le naufrage ou par les corsaires, si ce n'est $q$ 'on ait pris une assûrance. ${ }^{\text {"17 }}$

17 Diderot (Anm. 16), S. 869a (Hervorhebung von mir, M. S.). 
Bereitgestellt von | UZH Hauptbibliothek / Zentralbibliothek Zürich Angemeldet Heruntergeladen am | 07.12.17 16:56 